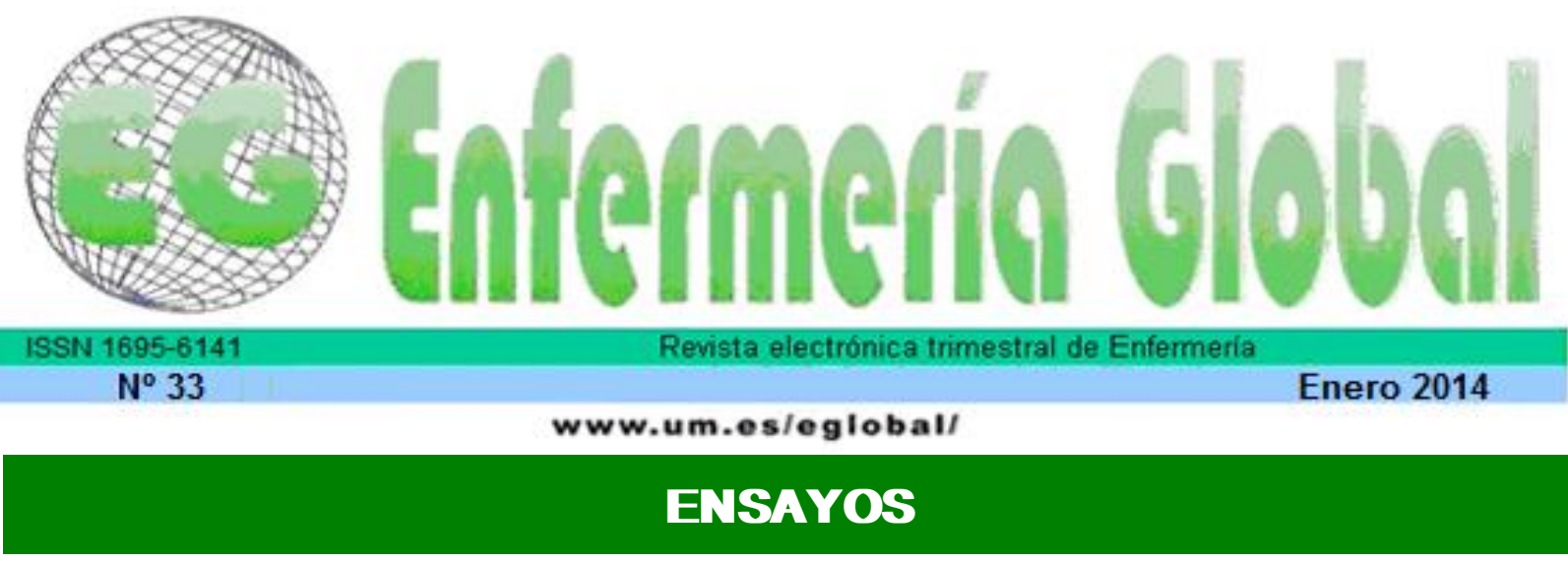

\title{
Enfermería desde la perspectiva del Trabajo Decente
}

Nursing from the perspective of Decent Work

\section{${ }^{*}$ Quintana Zavala, María Olga ${ }^{* *}$ Valenzuela Suazo, Sandra ***Paravic Klijn, Tatiana}

\author{
*Doctora en Enfermería. Profesora Titular del Departamento de Enfermería. Universidad de Sonora. \\ E-mail: mariaoquintana@udec.cl **Doctora. en Enfermería, Profesora Titular, Departamento de \\ Enfermería. Facultad de Medicina. Universidad de Concepción, Chile.
}

Palabras clave: Enfermería; Riesgos Laborales; Trabajo Decente.

Keywords: Nursing; Occupational Risks; Decent Work.

\section{RESUMEN}

Introducción: La Organización Internacional del Trabajo es un organismo que realiza propuestas y recomendaciones enfocadas a mejorar la calidad de vida de las y los trabajadores, evidenciando las condiciones deficientes de trabajo y enfatiza sobre la importancia de que todo ser humano tenga acceso a un Trabajo Decente, el cual es un concepto con profundos marcos éticos que busca la justicia social.

Objetivo: Reflexionar sobre las implicancias del trabajo de Enfermería en relación al Concepto de Trabajo Decente.

Desarrollo: El término Trabajo Decente surge como respuesta a la situación de creciente desprotección de los trabajadores(as), principalmente de los jóvenes, las mujeres y los más pobres. La Enfermería como profesión conformada en su mayoría por mujeres, se enfrenta a múltiples riesgos laborales, que la convierten en la profesión sanitaria con mayores riesgos a la salud, pero no en la mejor remunerada, ni en la más reconocida socialmente alrededor del mundo.

Consideraciones finales: El Trabajo Decente implica salarios competitivos, seguridad social, globalización equitativa, formación, equidad de género, libre de riesgos, con derechos laborales e igualdad. Los profesionales de Enfermería, las Instituciones de Salud y los Gobiernos, deben reconsiderar las condiciones de trabajo y los múltiples riesgos adicionados a la labor de las Enfermeras, para hacer que la Enfermería cumpla con todas las características de un trabajo decente, fomentando la prevención de riesgos laborales e incentivando con mejores salarios y condiciones de trabajo en general, para mejorar la calidad de vida laboral de las Enfermeras y Enfermeros. 


\section{ABSTRACT}

Introduction: The International Labor Organization encourages improvement in the quality of life for workers, highlighting poor working conditions and promoting Decent Work for all.

Objective: To reflect on the implications of the work of nursing in relation to the concept of Decent Work.

Development: Decent Work responds to the increasing vulnerability of workers, mainly young people, women and the poor. Nursing as a profession is made up mostly of women, faces multiple occupational hazards, including psychosocial risk factors, biological, physical, chemical and ergonomic, which means that this health care profession has higher health risks, is not the best paid job, nor is well recognized socially around the world.

Conclusion: Decent Work involves competitive salaries, social security, fair globalization, education, gender equality, low risk, with labor rights and fairness. Nursing professionals, health institutions and governments should reconsider attending to the multiple risks that are faced in any hospital to make nursing work comply with all the features of Decent Work, promoting the reduction of occupational hazards and providing better salaries and working conditions in general to all nurses and improving the quality of their working life.

\section{INTRODUCCIÓN}

La Organización Internacional del Trabajo (OIT) fue creada, como parte del Tratado de Versalles en 1919 para que gobiernos, empleadores y trabajadores unieran sus esfuerzos en pro de la justicia social y de mejores condiciones de vida y empleo, para todos los seres humanos ${ }^{1}$.

La OIT a través de la historia ha realizado propuestas y recomendaciones enfocadas a mejorar la calidad de vida de los trabajadores y trabajadoras, evidenciando las injusticias a las que se exponen por la necesidad de tener un empleo y ha destacado la importancia de que todo ser humano tenga acceso a un Trabajo Decente (TD), lo que implica empleo en cantidad y calidad suficiente, apropiado, digno y justo, que incluye el respeto de los derechos, ingresos adecuados y equitativos, condiciones de trabajo satisfactorias, protección social, en un contexto de libertad sindical y diálogo social $^{2}$.

Sin embargo, la realidad mundial en relación a la falta de empleos refleja una gran inseguridad en la que lamentablemente los más desprotegidos son las mujeres, los niños y niñas, los adultos mayores, los indígenas y/o las personas con alguna discapacidad, a los que no solo su condición les adjudica un grado de vulnerabilidad, sino que sumado a la situación de vivir en países en vías de desarrollo, zonas rurales, ser migrantes, no contar con escolaridad y/o ser de raza negra, hace más difícil el acceso a los recursos y específicamente a la oportunidad de que el ingreso familiar sea avalado por un TD. Aunado a lo anterior, las transformaciones laborales producto de la globalización, precarizan aún mas las condiciones de trabajo de los grupos vulnerables, por lo que es preciso enfatizar en la importancia de la formación profesional, el respeto a los derechos y dignidad de los trabajadores y trabajadoras, los que constituyen, características del TD ${ }^{3}$.

Enfermería, como grupo profesional, inserto en el mercado laboral, no está exento en los cambios de las condiciones laborales actuales y al realizar un análisis de algunas de las características del trabajo de Enfermería se podría decir que no se articula con el concepto de TD proporcionado por la OIT, ya que algunas de las formas de trabajo 
y contratación de enfermeras y enfermeros repercuten en su salud física y mental, lo que trasciende a la atención que brindan, situación que es necesario considerar ya que los Profesionales de Enfermería constituyen un gremio numeroso que da soporte a los servicios de salud alrededor del mundo.

Por lo anterior, el objetivo de este artículo es reflexionar sobre las implicancias del Trabajo de Enfermería en relación al concepto de TD proporcionado por la OIT, a través del desarrollo de los antecedentes históricos, la explicación del concepto, el análisis de las transformaciones en las relaciones laborales actuales y los desafíos que el TD representa para la Profesión de Enfermería.

\section{ANTECEDENTES HISTÓRICOS DE LA OIT}

La Organización Internacional del Trabajo (OIT) nace como parte del Tratado de Versalles, al final de la primera guerra mundial. "Su Constitución fue elaborada entre enero y abril de 1919 por una Comisión del Trabajo establecida por la Conferencia de Paz, que se reunió por primera vez en París y luego en Versalles. La Comisión, presidida por Samuel Gompers, presidente de la Federación Estadounidense del Trabajo, estaba compuesta por representantes de nueve países: Bélgica, Cuba, Checoslovaquia, Francia, Italia, Japón, Polonia, Reino Unido y Estados Unidos. El resultado fue una organización tripartita, la única en su género con representantes de gobiernos, empleadores y trabajadores en sus órganos ejecutivos" 4 .

La razón principal que motivó el surgimiento de la OIT se relacionó con la necesidad de adoptar regímenes de trabajo realmente humanos, en cuanto a: Reglamentación de las horas de trabajo, contratación de mano de obra, prevención del desempleo y suministro de un salario digno, protección del trabajador contra enfermedades 0 accidentes como consecuencia de su trabajo y de niños, jóvenes y mujeres, pensión de vejez e invalidez, protección de los intereses de los trabajadores ocupados en el extranjero, reconocimiento de los principios de igualdad de retribución en igualdad de condiciones y de libertad sindical y organización de la enseñanza profesional y técnica ${ }^{4}$.

Actualmente, el chileno Juan Somavia es el noveno Director General de la OIT, quien asumió su mandato de cinco años el 4 de marzo de 1999, convirtiéndose así en el primer representante del hemisferio sur en dirigir la OIT. En marzo de 2003, fue reelegido para un segundo mandato de cinco años, y para un tercer mandato el 18 de noviembre de 2008 y fue quien propuso el Programa de Trabajo Decente, el cual ha impulsado durante su gestión ${ }^{5}$.

\section{CONCEPTO DE TRABAJO DECENTE}

El concepto de TD nace como respuesta a la creciente desprotección de los trabajadores y trabajadoras y a las situaciones de inseguridad en que se desenvuelven las sociedades contemporáneas, ante la problemática mundial en torno a la deshumanización del trabajo y a la tendencia neoliberal que prefiere la producción y la generación de riquezas y como consecuencia, la explotación de la persona humana, especialmente en los países en vías de desarrollo, es decir, en los países en los que existe un retraso en términos de desarrollo humano, medido a través de tres indicadores: vida larga y saludable, educación y nivel de vida digno ${ }^{6}$. 
La OIT declara que el TD es: "el trabajo productivo en el cual los derechos son respetados, con seguridad y protección y con la posibilidad de participación en las decisiones que afectan a los trabajadores", además, el TD incorpora los siguientes atributos: empleo de calidad y cantidad suficiente, ingresos adecuados, seguridad en el trabajo, formación profesional continua y pertinente a la empleabilidad, respeto a los derechos de los trabajadores y trabajadoras, fortalecimiento sindical y negociación colectiva, participación de los trabajadores en las decisiones de política económica y social, diálogo social y tripartismo, protección social en el empleo y en la sociedad, en condiciones de libertad, equidad y dignidad para todos los miembros de la sociedad ${ }^{6}$.

Algunos autores argumentan que el concepto de TD es un término aún en construcción, debido a las constantes transformaciones de las relaciones laborales, la competitividad perseguida por las empresas, tanto en los sectores de punta como en los tradicionales, lo que ha puesto de relieve la aparición de nuevas formas de organización productiva, entre las cuales se encuentran: los grupos de empresas, el arrendamiento de servicios, el trabajo a domicilio y diversas formas de aglutinar la gestión externa a las obras o servicios mediante contratos auxiliares conocidos comúnmente como "outsourcing", que se traduce como "externalizar", lo que se refiere a la subcontratación de servicios profesionales para satisfacer necesidades específicas y disminuir los costos de contratación que ha llevado a modelos diferenciados de producción que ocasionan problemas a la configuración del derecho del trabajo y seguridad social ${ }^{7}$.

EI TD provoca debates en la forma de su operacionalización ya que se pretende que sea una realidad en todo el mundo, los debates se vigorizan cuando se refieren a los países con mayores índices de pobreza, lo que constituye una crítica fuerte ante el

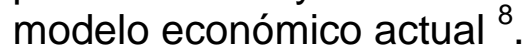

\section{LAS TRANSFORMACIONES EN LAS RELACIONES LABORALES}

Las transformaciones en las relaciones laborales producto de la globalización, constituyen aspectos tales como: Cambios en el empleo; Flexibilización en el mercado de trabajo; Cambios en la normativa laboral; Reestructuración de los sistemas de seguridad social; Debilitamiento del sindicalismo y de las negociaciones colectivas; Debilitamiento del ol del Estado como asignador de recursos y como agente redistributivo del ingreso, y Fortalecimiento de la autonomía empresarial no sólo para la toma de decisiones económicas, sino también para definir unilateralmente las condiciones de trabajo y de salarios y los márgenes de rentabilidad que quieren alcanzar para sus negocios ${ }^{6}$.

Para mitigar algunas de las transformaciones laborales negativas, el principal objetivo de la OIT es "promover estrategias para que mujeres y hombres puedan obtener un TD y productivo, en condiciones de libertad, igualdad, seguridad y dignidad humana", a través del poner en práctica sus cuatro objetivos estratégicos, que son: 1) Crear trabajo; 2) Garantizar los derechos de los trabajadores; 3) Extender la protección social; 4) Fortalecer el tripartismo y promover el dialogo social, con equidad de género ${ }^{9}$.

Mientras que en el mundo, existe un déficit de TD, por lo que es urgente que los gobiernos asuman el desafío de crear 600 millones de puestos de trabajo productivos en el próximo decenio y aun así quedarán 900 millones de trabajadores que viven con sus familias con ingresos inferiores al umbral de la pobreza de los 2 dólares de 
Estados Unidos por día, sobre todo en países en vías de desarrollo. El panorama de la creación de empleos afecta especialmente a los jóvenes, ya que existen 74.8 millones de jóvenes de entre 15 y 24 años desempleados. Entre los 900 millones de trabajadores pobres en el mundo, 456 millones viven en pobreza extrema con ingresos inferiores al umbral de 1.25 dólares. A nivel mundial, se estima en 1.520 millones el número de trabajadores en situación de vulnerabilidad en el empleo. La proporción de mujeres en situación de vulnerabilidad laboral es del $50.5 \%$ y excede a la de los hombres ${ }^{3}$.

En América Latina, la perspectiva es positiva, ya que tras la interrupción provocada por la crisis mundial en 2009, la tasa de empleo vulnerable continúa descendiendo y según las tendencias a largo plazo las mujeres adultas continuarán beneficiándose de oportunidades de empleo ${ }^{3}$, sin embargo, para que este empleo sea considerado como TD es de gran importancia que cumplan con condiciones laborales adecuadas, derechos laborales y apoyo a la formación profesional, puesto que, como declara el ganador del Premio Nobel de Economía Amartya Sen: "no habrá trabajo decente sin justicia social y ciudadanía. Y no habrá nada de esto sin educación, incluida la formación profesional" ${ }^{10}$.

\section{DESAFÍOS DEL TRABAJO DECENTE PARA ENFERMERÍA}

Al analizar algunas de las características del trabajo de Enfermería bajo el concepto del TD, se puede afirmar que hay puntos a considerar dentro de la labor de las enfermeras y enfermeros, alrededor del mundo, que no se articulan con el concepto, entre ellos, lo relacionado a los diversos riesgos que presenta el trabajar en áreas de hospital, entendiéndose como riesgo a cualquier característica presente en el ambiente laboral que al actuar sobre el trabajador puede producir una respuesta, de incomodidad, dolor o lesión ${ }^{11}$. Ya que existe evidencia de que el trabajo que realizan los profesionales de Enfermería dentro de los hospitales es el que tiene más factores de riesgo a la salud, comparado con otros grupos de profesionales sanitarios. Los riesgos mas importantes son los: psicosociales, biológicos, físico-químicos y ergonómicos ${ }^{12,13}$.

Aunado a lo anterior, Enfermería como profesión feminizada, sigue presentando discriminación de género, lo que se refleja en la subvaloración laboral, salarios menores a otros profesionales sanitarios y alto cuestionamiento de su actividad profesional $^{14}$.

Asimismo, está documentado que dentro del equipo de Enfermería existe desconfianza de Enfermeras hacia sus jefaturas, principalmente por maltrato y falta de apoyo en relación a permisos solicitados ante situaciones especiales, lo que repercute en el liderazgo, el trabajo en equipo, el clima laboral y perjudica el adecuado funcionamiento de los servicios de salud ${ }^{15}$.

Por otra parte, el trabajo de las enfermeras y enfermeros, ha sido alcanzado por algunas de las transformaciones económicas producto de la globalización, ya que aunque es una profesión que tiene alta demanda en la mayoría de los países, es cada vez más común que se presenten contrataciones eventuales en instituciones públicas y/o privadas, lo que repercute en la estabilidad laboral y obstaculiza a las enfermeras y enfermeros a reclamar sus derechos, debido a que en ocasiones el hecho de ser contratadas(os) de forma eventual o indeterminada, imposibilita el acceso en algunos casos a prestaciones laborales básicas, como ingresar a sindicatos, períodos 
vacacionales, incapacitarse en caso de enfermedad, acceder a educación continua, concursar para ascensos, servicios de salud, entre otras, además las eventualidades son de larga duración, ya que existe poca creación de plazas nuevas.

En situación más difícil se encuentran los Profesionales de Enfermería, en algunos países centroamericanos, ya que es evidente la falta de empleos, mientras que en otros países de América Latina el trabajo existe, pero los salarios son menores a los de otros profesionales del área de la salud y las condiciones en las que se desempeñan no son adecuadas; en cuestión de derechos laborales, la conformación de sindicatos es todavía una práctica aceptada en algunos países, pero la tendencia es a desaparecerlos, sobre todo en las instituciones privadas de salud, en donde conformarlos llega a ser motivo de despido; en cuanto a la protección social, la tendencia es a desproteger a los trabajadores con contrataciones externas, situación que ha alcanzado a Enfermería.

En el caso de México, la tasa global de desperdicio laboral de licenciados en Enfermería, (proporción de personas que concluyeron la licenciatura y no la ejercen) es de 410 por cada 1000 personas $^{16}$, mientras que la migración de enfermeras profesionales a países como España, Canadá y Estados Unidos, es un fenómeno que va en aumento, lo cual tiene impacto tanto de índole social, como económico, ya que tiene efectos negativos en la salud de la población, por constituir el grupo de profesionales sanitarios que logran una mayor cobertura de atención, sin embargo, las principales razones identificadas para desear migrar, son los salarios bajos y la falta de estatus profesional o poca valoración de la profesión por la sociedad ${ }^{17}$.

Sin embargo, la problemática del trabajo en los profesionales de enfermería es alarmante también en los países industrializados ya que la remuneración en los servicios de salud del sector público se ha deteriorado. "Las mujeres, que representan al $80 \%$ de los trabajadores del sector, se sitúan en las escalas jerárquicas más bajas en cuanto a autoridad, retribución y cualificación", sumado a lo anterior, casi una cuarta parte de los incidentes violentos en el trabajo se concentra en este sector ${ }^{17}$.

El enfoque de la profesión de Enfermería coincide con la OIT, ya que el Trabajo de Enfermería no puede ser considerado como una mercancía, la libertad de expresión y de asociación de las Enfermeras y Enfermeros es esencial para el progreso de la Profesión, la pobreza en cualquier lugar es una amenaza para la prosperidad de todos, los seres humanos sin distinción de raza, credo o sexo, tienen derecho a perseguir su bienestar material y su desarrollo espiritual, en condiciones de libertad y dignidad, de seguridad económica y en igualdad de oportunidades.

\section{CONSIDERACIONES FINALES}

La Enfermería como grupo profesional del área de la salud constituye el recurso humano más numeroso y desempeña acciones muy importantes, entre las cuales destacan proveer cuidado profesional, ampliar la cobertura de los servicios de salud, contribuir a la mejora en la salud pública de las poblaciones, además de estar en contacto directo de forma permanente con los usuarios y usuarias.

Por lo anterior y al igual que otras profesiones sanitarias, el trabajo de Enfermería debe de cumplir con las características del TD, lo que implica el acceso a: salarios justos, seguridad social, formación profesional, equidad de género, libre de riesgos y derechos laborales en igualdad de circunstancias para todas y todos. 
Los profesionales de Enfermería, las Instituciones de Salud y los Gobiernos, deben de tomar en cuenta, los múltiples riesgos adicionados al trabajo de Enfermería en el área de hospitales, fomentando la prevención de riesgos laborales e incentivando a Enfermeras con mejores salarios y mejores condiciones de trabajo en general.

La labor de Enfermería debe enfocarse también en promover a nivel de políticas públicas cambios estructurales que propicien el TD para enfermeras y enfermeros y para todos los trabajadores. Los gobiernos, las instituciones de salud y los Profesionales de Enfermería, deben de reconocer que el Trabajo de Enfermería es sumamente valioso para la salud de las poblaciones, por lo que es necesario desarrollar estrategias que propicien mejores ambientes laborales, que promuevan, además, la disminución de riesgos y que motiven a ingresar y permanecer en el ejercicio de la profesión.

\section{REFERENCIAS BIBLIOGRÁFICAS}

(1) Organización Internacional del Trabajo. Acerca de la OIT. [Internet]. [Consultado el día 15 de Abril del 2012] Disponible en: http://www.ilo.org/global/about-the-ilo/lang-es/index.htm

(2) Méndez C. Por un desarrollo equitativo, inclusivo y sostenible. Trabajo Decente. [Internet]. [Consultado el día 15 de Abril del 2012] Disponible en: http://www.desarrollosustentable.com.ve/site/index.php?view=items\&cid=18\%3Aexper iencias-e-investigaciones-sociales\&id=190\%3Atrabajo-decente-charomacndez\&format=pdf\&option=com flexicontent\&ltemid $=19$

(3) OIT. Tendencias mundiales del empleo 2012. Prevenir una crisis mayor del empleo. Oficina Internacional del Trabajo. Ginebra [Internet]. [Consultado el día 15 de Abril del 2012] Disponible en:http://www.ilo.org/wcmsp5/groups/public/---dgreports/--dcomm/-- publ/documents/publication/wcms 168095.pdf

(4) OIT. Orígenes e historia. [Internet]. [Consultado el día 15 de Abril del 2012] Disponible de http://www.ilo.org/global/about-the-ilo/history/lang--es/index.htm

(5) OIT. Biografía del Director General Juan Somavia. [Internet]. [Consultado el día 15 de Abril del 2012] Disponible en: http://www.ilo.org/public/spanish/bureau/dgo/biography.htm

(6) Espinoza M. Trabajo decente y protección social. Santiago, Oficina Internacional del Trabajo, 2003. ISBN 92-2-314231-8

(7) Walker F. Rol de los Estados para hacer cumplir las normas internacionales del trabajo y promover el trabajo decente. Rev Lab Chil. 2005; 89-99

(8) OIT. Las reuniones internacionales destacan el creciente "movimiento global a favor del trabajo decente". Trabajo [Internet] 2008; 65:60-61 [Consultado el día 15 de Abril del 2012] Disponible en: http://international.vlex.com/vid/decente-reunionesdestacan-54095238

(9) OIT. Misión y Objetivos. [Internet]. [Consultado el día 15 de Abril del 2012] Disponible en: http://www.ilo.org/global/about-the-ilo/mission-and-objectives/lang-es/index.htm

(10) OIT. Cinterfor. Trabajo decente y formación profesional. Montevideo 2001. [Internet].[Consultado el 18 de abril del 2012]. Disponible de http://www.ilo.org/public//spanish/region/ampro/cinterfor/publ/boletin/153/index.htm

(11) Ministerio del Trabajo y Previsión Social. Guía Técnica para la evaluación y control de los riesgos asociados al manejo o manipulación manual de carga [Internet]. Santiago: Gobierno de Chile; 2008. [Consultado el 18 de abril del 2012]. Disponible en: http://www.cec.uchile.cl/ com-parit/images/comite/guiacarga.pdf 
(12) Carvallo B. Riesgos laborales en el ejercicio de la enfermería. Actual. Enferm. 2003; 6 (3):25-30.

(13) Mateu N, Patricio B, Farré C, Mas M. Protegiéndonos ganamos en salud y ahorramos en recursos. Paraninfo digital. 2011; 12.

(14) "No perjudicar". El dialogo social también beneficia a los pacientes. Trabajo. [Internet] 2004; 51:19-20. [Consultado 2012 Julio 16]. Disponible en: http://www.ilo.org/wcmsp5/groups/public/@dgreports/@dcomm/documents/publication /dwcms 080712.pdf

(15) Yañez-Gallardo R, Valenzuela-Suazo S. Incidentes críticos de erosión de la confianza en el liderazgo de enfermería. Rev. Latino-Am. Enfermagem [Internet]. 2012 [Consultado el día 5 de Agostodel2012]; 20(1):143-150.Disponible en: http://www.scielo.br/scielo.php?script=sci_arttext\&pid=S010411692012000100019\&ln $\mathrm{g}=$ en. http://dx.doi.org/10.1590/S0104-11692012000100019.

(16) Nigenda G, Ruiz JA, Rosales Y, Bejarano R. Personal de enfermería con licenciatura en México: valoración del desperdicio en su formación y en el mercado de trabajo. Salud Pública Mex 2006; 48:22-9.

(17) Rosales-Martínez Y, Nigenda G, Galarraga O, Ruiz-Larios JA. Expectativas de migración internacional en estudiantes de enfermería en México, Distrito Federal. Salud pública Méx [Internet]. 2010; 52(3): 244-53 [Consultado el 5 de Agosto del 2012].

Disponible en:

<http://www.scielo.org.mx/scielo.php?script=sci_arttext\&pid=S0036-

36342010000300009\&Ing=es\&nrm=iso >. ISSN 0036-3634. 